\title{
Pseudocomplementation in (normal) subgroup lattices
}

\author{
Tom De Medts Marius Tărnăuceanu
}

October 21, 2009

\begin{abstract}
The goal of this paper is to study finite groups admitting a pseudocomplemented subgroup lattice (PK-groups) or a pseudocomplemented normal subgroup lattice (PKN-groups). In particular, we obtain a complete classification of finite PK-groups and of finite nilpotent PKN-groups. We also study groups with a Stone normal subgroup lattice, and we classify finite groups for which every subgroup has a Stone normal subgroup lattice. Finally, we obtain a complete classification of finite groups for which every subgroup is monolithic.
\end{abstract}

MSC2000: primary 06D15, 20D30; secondary 20D15, 20D20

Key words: pseudocomplement, subgroup lattice, normal subgroup lattice, monolithic group, Stone lattice

\section{Introduction}

It is an interesting question in group theory in how far the structure of the subgroup lattice of a group determines the structure of the group itself. This question in its pure form is quite old [11,2], and M. Suzuki spent his early research years on this problem $[14,15]$. Since then, many characterizations and classifications have been obtained for groups for which the subgroup lattice or normal subgroup lattice has certain lattice-theoretic properties. The possibly most famous result in this direction is Ore's result that a group 
is locally cyclic if and only if its lattice of subgroups is distributive [9]. We refer to Suzuki's book [17], Schmidt's book [12] or the more recent book [23] by the second author for more information about this theory.

In the current paper, we investigate (finite) groups which have a subgroup lattice or normal subgroup lattice which is pseudocomplemented; these are called PK-groups and PKN-groups, respectively. We obtain a complete classification of finite PK-groups and of finite nilpotent PKN-groups, thereby vastly improving earlier results $[5,19]$. We will also classify groups with the property that each subgroup is itself a PKN-group, and we will give some results about groups for which the normal subgroup lattice is a so-called Stone lattice. In particular, we will classify finite groups for which the normal subgroup lattice of every subgroup is a Stone lattice, and as a corollary, we obtain a complete classification of finite groups for which every subgroup is monolithic.

Let $(L, \wedge, \vee)$ be a bounded lattice with top 1 and bottom 0 , and let $a \in L$. An element $b \in L$ is called a complement of $a$ if $a \vee b=1$ and $a \wedge b=0$, and the lattice $L$ is called complemented if every element of $L$ has a complement. An element $a^{*} \in L$ is called a pseudocomplement of $a$ if the following two conditions are satisfied:

(i) $a \wedge a^{*}=0$;

(ii) $a \wedge x=0(x \in L)$ implies $x \leq a^{*}$.

Any element of $L$ can have at most one pseudocomplement. We say that $L$ is a pseudocomplemented lattice if every element of $L$ has a pseudocomplement. Note that the terminology is slightly misleading, since a complement is not necessarily a pseudocomplement; in fact, a complement of an element need not be unique.

In a pseudocomplemented lattice $L$ the set $S(L)=\left\{x^{*} \mid x \in L\right\}$ forms a lattice (called the skeleton of $L$ ), which is a $\wedge$-subsemilattice of $L$ and in which the join is defined by $x \sqcup y=(x \vee y)^{* *}=\left(x^{*} \wedge y^{*}\right)^{*}$. The lattice $(S(L), \wedge, \sqcup)$ is in fact a boolean lattice [7, Theorem I.6.4]; this result was first proved for complete distributive lattices by V. Glivenko [6].

A lattice $L$ is called distributive if the identity

$$
a \wedge(b \vee c)=(a \wedge b) \vee(a \wedge c)
$$


holds for all $a, b, c \in L$. A pseudocomplemented distributive lattice $L$ for which the skeleton $S(L)$ is a sublattice of $L$ is called a Stone lattice.

Let $(G, \cdot 1)$ be a group (where 1 denotes the identity of $G$ ). Then the set $L(G)$ consisting of all subgroups of $G$ is a complete bounded lattice with top $G$ and bottom $\{1\}$, called the subgroup lattice of $G$. The normal subgroup lattice $N(G)$ of $G$ is the modular sublattice of $L(G)$ constituted by all normal subgroups of $G$. Many classes of groups determined by different properties of their subgroup lattices or normal subgroup lattices (such as modularity, distributivity, complementation,... and so on) have been identified. In the present paper our aim is to investigate the groups $G$ for which the lattice $L(G)$ or $N(G)$ is pseudocomplemented. Observe that if $G$ is a group such that $L(G)$ is pseudocomplemented, then for every subgroup $H \leq G$, the pseudocomplement $H^{*}$ is the unique subgroup that is maximal amongst those that intersect $H$ trivially; a similar observation holds for $N(G)$.

The paper is organized as follows. In Section 2 we study the class of finite PK-groups, i.e. the groups with pseudocomplemented subgroup lattice. In Section 3 we look at finite PKN-groups, i.e. the groups with pseudocomplemented normal subgroup lattice. Section 4 deals with groups for which all subgroups have pseudocomplemented lattices of normal subgroups. In the fi-

nal section 5, finite groups whose normal subgroup lattices are Stone lattices will be investigated.

Most of our notation is standard and will not be repeated here. Basic definitions and results on lattices and groups can be found in [3, 7] and [1, 18], respectively. For subgroup lattice concepts we refer the reader to [12, 17, 23].

We only mention that by a generalized quaternion group, we mean a group of order $2^{t}$ for some natural number $t$, defined by the presentation

$$
Q_{2^{t}}=\left\langle a, b \mid a^{2^{t-2}}=b^{2}, a^{2^{t-1}}=1, b^{-1} a b=a^{-1}\right\rangle
$$

and not the more general notion of groups of order $4 n$ of the form $Q_{4 n}=$ $\left\langle a, b \mid a^{n}=b^{2}, a^{2 n}=1, b^{-1} a b=a^{-1}\right\rangle$ which is used by some authors.

\section{PK-groups}

Definition 2.1. A group $G$ is called a $P K$-group if its lattice of subgroups $L(G)$ is pseudocomplemented, and it is called a PKN-group if its lattice of 
normal subgroups $N(G)$ is pseudocomplemented.

PK-groups were introduced and studied in [19] and [20]. Our goal in this section is to give a complete classification of finite PK-groups. For the sake of readability, we will include a complete proof, even though some parts of Lemma 2.3 and Proposition 2.5 have already appeared (in a different form) in [19] or [20].

Theorem 2.2. Let $G$ be a finite group of order $2^{t} m$ with $m$ odd. Then $G$ is a PK-group if and only if it is cyclic or it is isomorphic to the direct product of a cyclic group of order $m$ and a generalized quaternion group of order $2^{t}$.

We start with the following easy but important lemma.

Lemma 2.3. (i) Every subgroup of a PK-group is again a PK-group;

(ii) every cyclic group is a PK-group;

(iii) a group of order $p^{2}, p$ prime, is a PK-group if and only if it is cyclic;

(iv) every generalized quaternion group is a PK-group.

Proof. (i) Let $G$ be PK-group and let $H \leq G$. If $A$ is a subgroup of $H$ and the pseudocomplement of $A$ in $G$ is $A^{*}$, then $A^{*} \cap H$ is the pseudocomplement of $A$ in $H$.

(ii) Let $G$ be a finite cyclic group, and let $A$ be an arbitrary subgroup of $G$. Let $s$ be the largest divisor of $|G|$ coprime to $|A|$, and let $A^{*}$ be the unique subgroup of $G$ of order $s$. Then $A^{*}$ is the pseudocomplement of $A$ in $G$.

Now let $G$ be the infinite cyclic group $(\mathbb{Z},+)$; then any two non-trivial subgroups of $G$ intersect non-trivially. So let $A$ be an arbitrary subgroup of $G$, then $A^{*}=\{0\}$ is a pseudocomplement of $A$ in $G$.

(iii) Let $G$ be a group of order $p^{2}$, and assume that $G$ is not cyclic. Then $G \cong C_{p} \times C_{p}$, hence $G$ has $p+1$ subgroups of order $p$, any two of which intersect trivially. Take any one of them, say $A$; then every other of these subgroups is maximal amongst those intersecting $A$ trivially. Hence $G$ is not a PK-group.

(iv) Let $G$ be a generalized quaternion group. It is well known that such a group contains a unique involution $z$; see, for example, [1, Chapter 8, 
Exercise $3(7)]$. But then every non-trivial subgroup $A$ of $G$ contains $z$, and hence $A^{*}=1$ is a pseudocomplement for $A$ in $G$.

Corollary 2.4. Let $G$ be a finite PK-group. Then every Sylow subgroup of $G$ is either cyclic or generalized quaternion.

Proof. Let $p$ be any prime divisor of $|G|$, and let $S$ be a Sylow $p$-subgroup of $G$; by Lemma 2.3(i and iii), every subgroup of $S$ of odd order $p^{2}$ is cyclic. Equivalently, $S$ has a unique subgroup of order $p$, since the center $Z(S)$ of $S$ always contains a subgroup of order $p$. If $p$ is odd, this implies that $S$ is cyclic, and if $p=2$, this implies that $S$ is either cyclic or generalized quaternion; see, for example, [1, Chapter 8, Exercise 4].

Before we proceed, we show that the PK and PKN properties behave well with respect to direct products of groups of coprime order.

Proposition 2.5. Let $G \cong G_{1} \times \cdots \times G_{n}$ with $\operatorname{gcd}\left(\left|G_{i}\right|,\left|G_{j}\right|\right)=1$ for all $i, j \in\{1, \ldots, n\}$ with $i \neq j$.

(i) $G$ is a PK-group if and only if each $G_{i}$ is a PK-group;

(ii) $G$ is a PKN-group if and only if each $G_{i}$ is a PKN-group.

Proof. We only prove (ii); the proof of (i) is similar. So assume first that $G$ is a PKN-group, let $i \in\{1,2, \ldots, n\}$ and let $N \unlhd G_{i}$. Then $N \unlhd G$, hence $N$ has a pseudocomplement $N^{*}$ in $G$; it is clear that $N^{*} \cap G_{i}$ is a pseudocomplement of $N$ in $G_{i}$.

Conversely, assume that each $G_{i}$ is a PKN-group, and let $N \unlhd G$; since $\operatorname{gcd}\left(\left|G_{i}\right|,\left|G_{j}\right|\right)=1$ for all $i, j \in\{1, \ldots, n\}$ with $i \neq j$, we can write $N=$ $N_{1} \times \cdots \times N_{n}$, where $N_{i} \unlhd G_{i}$, for all $i \in\{1, \ldots, n\}$. For each $i$, let $N_{i}^{*}$ be a pseudocomplement of $N_{i}$ in $G_{i}$. Then $N^{*}=N_{1}^{*} \times \cdots \times N_{n}^{*}$ is a pseudocomplement of $N$ in $G$.

Remark 2.6. It is clear from Lemma 2.3(iii) that the coprimeness condition cannot be omitted.

We are now ready to prove Theorem 2.2. We are grateful to the anonymous referee of an earlier version of this article for the short argument; our original proof was much more involved. 
Proof of Theorem 2.2. Assume first that $G$ is either cyclic or isomorphic to the direct product of a cyclic group of odd order $m$ and a generalized quaternion group of order $2^{t}$. Then it is clear from Lemma 2.3(ii and iv) together with Proposition 2.5(i) that $G$ is a PK-group.

Assume now that $G$ is a PK-group. We will show that $G$ is nilpotent; the result will then follow immediately from Corollary 2.4 since a finite nilpotent group is the direct product of its Sylow subgroups.

So let $P$ be an arbitrary Sylow $p$-subgroup of $G$ for some prime divisor $p$ of $|G|$, and let $N$ be a pseudocomplement for $P$ in $G$. Moreover, let $R$ be the subgroup of $G$ generated by all $p^{\prime}$-elements of $G$; then $R$ is characteristic in $G$. Since $N$ contains every $p^{\prime}$-element of $G$, we have $R \leq N$. Because $N \cap P=1$, however, we have $R \cap P=1$ as well, and hence $R$ intersects every Sylow $p$-subgroup trivially. We conclude that $R$ is a Hall $p^{\prime}$-subgroup of $G$, and since it is normal, it is, in fact, a normal $p$-complement.

This holds for every prime divisor $p$ of $|G|$, and hence $G$ has a normal $p$-complement for each of its prime divisors. It follows that every Sylow subgroup of $G$ is normal (as it is the intersection of normal Hall subgroups), and thus $G$ is nilpotent.

\section{PKN-groups}

In contrast to the situation of PK-groups, a (normal) subgroup of a PKNgroup is not necessarily a PKN-group. For example, the dihedral group $D_{8}$ of order 8 is a PKN-group, but has a normal subgroup $C_{2} \times C_{2}$, which is not a PKN-group. This simple fact makes the study of PKN-groups considerably harder.

Some of the results in this section have already appeared in [19, 20, 21, 22], but we believe it is interesting to include them nevertheless, and the proofs are somewhat more polished than in loc. cit. More specifically, Proposition 3.5 was known, and the statement of Proposition 3.8 already appears in [22], but the proof which is given there is inaccurate.

We start with an easy but useful observation.

Proposition 3.1. Let $G$ be a finite PKN-group. Then the center $Z(G)$ of $G$ is cyclic. 
Proof. Let $H \leq Z(G)$ be arbitrary; then $H \unlhd G$, so it has a pseudocomplement $H^{*}$ in $N(G)$. It is clear that $Z(G) \cap H^{*}$ is a pseudocomplement of $H$ in $L(Z(G))$. Hence $Z(G)$ is a PK-group; the result now follows from Theorem 2.2 since $Z(G)$ is abelian.

Proposition 2.5(ii) shows that the PKN property behaves well with respect to direct products of coprime order. For arbitrary direct products, we still have the following facts.

Lemma 3.2. Let $G=G_{1} \times \cdots \times G_{n}$ be a PKN-group. Then

(i) each $G_{i}$ is a PKN-group;

(ii) if $N \unlhd G$ is such that $N \cap G_{i}=1$ for all $i$, then $N=1$.

Proof. (i) Let $i \in\{1,2, \ldots, n\}$ and let $N \unlhd G_{i}$. Then $N \unlhd G$, hence $N$ has a pseudocomplement $N^{*}$ in $G$; it is clear that $N^{*} \cap G_{i}$ is a pseudocomplement of $N$ in $G_{i}$.

(ii) Let $N^{*}$ be the pseudocomplement of $N$ in $G$. Since $N \cap G_{i}=1$, we have $G_{i} \leq N^{*}$ for all $i$, hence $N^{*}=G$ and since $N \cap N^{*}=1$ this implies $N=1$.

Remark 3.3. By a well-known result of J. Wiegold [26], a group has a complemented lattice of normal subgroups (such a group is called an $n D$ group - see [24]) if and only if it is a direct product of simple groups. So an $\mathrm{nD}$-group is not always a PKN-group. A sufficient condition for a direct product $G$ of simple groups to become a PKN-group is that no two its minimal abelian factors are isomorphic. In this case $N(G)$ is a boolean lattice (see [25, Lemma 1]) and hence a pseudocomplemented lattice.

In any normal subgroup lattice $N(G)$ we have $1^{*}=G$ and $G^{*}=1$. A special type of PKN-groups $G$ is obtained when the skeleton $S(N(G))$ only contains the subgroups 1 and $G$.

Definition 3.4. We say that a PKN-group $G$ is elementary if $S(N(G))=$ $\{1, G\}$.

A characterization of finite elementary PKN-groups is given by the following result. 
Proposition 3.5. A finite group $G$ is an elementary PKN-group if and only if it is a monolithic group, i.e. a group with a unique minimal normal subgroup.

Proof. Suppose that $G$ is an elementary PKN-group and assume that it has at least two minimal normal subgroups $M_{1}$ and $M_{2}$. Because $M_{1} \cap M_{2}=1$, it follows that $M_{2} \leq M_{1}^{*}$ and therefore $M_{1}^{*} \neq 1$, a contradiction. Conversely, suppose that $G$ is a monolithic group and let $M$ be its unique minimal normal subgroup. Then for every $H \unlhd G$ with $H \neq 1$, we have $M \leq H$. This shows that $H^{*}=1$. Hence $G$ is an elementary PKN-group.

Remark 3.6. This result is no longer true for infinite groups, since an infinite group may have no minimal normal subgroups at all. For example, the infinite cyclic group $(\mathbb{Z},+)$ is elementary PKN, but has no minimal normal subgroups.

Remark 3.7. Using Proposition 3.5, many classes of elementary PKNgroups can be obtained. For example, any group whose normal subgroup lattice is a chain is an elementary PKN-group. In particular, simple groups, symmetric groups, cyclic $p$-groups or finite groups of order $p^{n} q^{m}(p, q$ distinct primes) with cyclic Sylow subgroups and trivial center [12, Exercise 3, p. 497] are all elementary PKN-groups; see also Theorem 5.9 and Theorem 5.12 below. We also mention that any proper semidirect product of type $G=\langle a\rangle M$, where $M$ is a maximal normal subgroup of $G$ having a fully ordered lattice of normal subgroups, is an elementary PKN-group.

Obviously, elementary PKN-groups $G$ have the property that the lattice $S(N(G))$ is a sublattice of $N(G)$. Our next result gives a characterization of finite PKN-groups for which this property holds.

Proposition 3.8. For a finite PKN-group $G$, the following two properties are equivalent:

(a) $S(N(G))$ is a sublattice of $N(G)$;

(b) $G$ is a direct product of elementary PKN-groups.

Proof. For each normal subgroup $N \unlhd G$, we will denote its pseudocomplement in $G$ by $N^{*}$. We claim that (a) is equivalent with the condition

$$
(A \cap B)^{*}=A^{*} B^{*} \text { for all } A, B \in S(N(G)) .
$$


Indeed, observe that $S(N(G))=\left\{A \unlhd G \mid A^{* *}=A\right\}$. If $S(N(G))$ is a sublattice of $N(G)$, then for all $A, B \in S(N(G))$, we have $A B=A \sqcup B=$ $\left(A^{*} \cap B^{*}\right)^{*}$, so by replacing $A$ with $A^{*}$ and $B$ with $B^{*}$ we obtain (3.1). Conversely, if (3.1) holds, then for all $A, B \in S(N(G))$, we have $A B=$ $A^{* *} B^{* *}=\left(A^{*} \cap B^{*}\right)^{*} \in S(N(G))$, so $S(N(G))$ is a sublattice of $N(G)$.

$(\mathrm{a}) \Rightarrow(\mathrm{b})$. To prove (b), we will use induction on $|G|$. Let $M_{1}, M_{2}, \ldots, M_{s}$ be the minimal normal subgroups of $G$. If $s=1$, then $G$ itself is an elementary PKN-group, so we may assume $s \geq 2$. Then $M_{1}^{*} \cap M_{1}^{* *}=1$, and by equation (3.1),

$$
G=1^{*}=\left(M_{1}^{*} \cap M_{1}^{* *}\right)^{*}=M_{1}^{* *} M_{1}^{* * *}=M_{1}^{* *} M_{1}^{*},
$$

hence $G$ is the direct product of $M_{1}^{* *}$ and $M_{1}^{*}$. In particular, every minimal normal subgroup of $M_{1}^{* *}$ is also a minimal normal subgroup of $G$.

Now $M_{i} \leq M_{1}^{*}$, for all $i \in\{2, \ldots, s\}$, hence $M_{i} \cap M_{1}^{* *}=1$. Therefore $M_{1}^{* *}$ has a unique minimal normal subgroup, namely $M_{1}$, and hence $M_{1}^{* *}$ is an elementary PKN-group.

By Lemma 3.2(i), $M_{1}^{*}$ is again a PKN-group. It remains to show that $S\left(N\left(M_{1}^{*}\right)\right)$ is a sublattice of $N\left(M_{1}^{*}\right)$, since the result will then follow by the induction hypothesis. We have

$$
S\left(N\left(M_{1}^{*}\right)\right)=\left\{K^{*} \cap M_{1}^{*} \mid K \in N(G)\right\}=\left\{A \cap M_{1}^{*} \mid A \in S(N(G))\right\} .
$$

For any two elements $A \cap M_{1}^{*}$ and $B \cap M_{1}^{*}$ of $S\left(N\left(M_{1}^{*}\right)\right)$, we have

$$
\begin{aligned}
\left(A \cap M_{1}^{*}\right) \cap\left(B \cap M_{1}^{*}\right) & =(A \cap B) \cap M_{1}^{*} \in S\left(N\left(M_{1}^{*}\right)\right), \\
\left(A \cap M_{1}^{*}\right)\left(B \cap M_{1}^{*}\right) & =(A B) \cap M_{1}^{*} \in S\left(N\left(M_{1}^{*}\right)\right),
\end{aligned}
$$

where we have used the fact that $S(N(G))$ is a distributive lattice. This shows that $S\left(N\left(M_{1}^{*}\right)\right)$ is a sublattice of $N\left(M_{1}^{*}\right)$, as claimed.

(b) $\Rightarrow(\mathrm{a})$. Let $G=G_{1} \times \cdots \times G_{n}$, where each $G_{i}$ is an elementary PKNgroup. Let $K \in S(N(G))$ be arbitrary, and let $i \in\{1, \ldots, n\}$. Since $K \cap G_{i} \in S\left(N\left(G_{i}\right)\right)=\left\{1, G_{i}\right\}$, we have either $K \cap G_{i}=1$ (and then $\left.G_{i} \leq K^{*}\right)$ or $G_{i} \leq K$ (and then $K^{*} \cap G_{i}=1$ ). Let

$$
\begin{aligned}
& X=\left\{i \in\{1, \ldots, n\} \mid K \cap G_{i}=1\right\}=\left\{i \in\{1, \ldots, n\} \mid G_{i} \leq K^{*}\right\}, \\
& Y=\left\{i \in\{1, \ldots, n\} \mid K^{*} \cap G_{i}=1\right\}=\left\{i \in\{1, \ldots, n\} \mid G_{i} \leq K\right\},
\end{aligned}
$$


and write $A=\prod_{i \in X} G_{i}$ and $B=\prod_{i \in Y} G_{i}$. Then $G=A \times B$; by Lemma 3.2(i), both $A$ and $B$ are PKN-groups. Observe that $B \leq K$. By the modular law,

$$
K=K \cap G=K \cap(A B)=B(K \cap A) .
$$

However, by Lemma 3.2(ii) applied on the PKN-group $A$, we have $K \cap A=1$. Hence $K=B=\prod_{i \in Y} G_{i}$.

Now take $K_{1}, K_{2} \in S(N(G))$; then there are subsets $Y$ and $Z$ of $\{1, \ldots, n\}$ such that $K_{1}=\prod_{i \in Y} G_{i}$ and $K_{2}=\prod_{i \in Z} G_{i}$. Since $S(N(G))$ is always a $\wedge$-subsemilattice of $N(G)$, we have $K_{1} \cap K_{2} \in S(N(G))$ (in fact $K_{1} \cap K_{2}=\prod_{i \in Y \cap Z} G_{i}$ ). On the other hand, it is obvious that $K_{1} K_{2}=\prod_{i \in Y \cup Z} G_{i} \in S(N(G))$. Hence $S(N(G))$ is a sublattice of $N(G)$.

Remark 3.9. If the equivalent properties of Proposition 3.8 are satisfied, then $S(N(G))$ is in fact a direct product of chains of length 1 ; this follows from the proof of $(\mathrm{b}) \Rightarrow(\mathrm{a})$.

A complete classification of finite PKN-groups seems to be out of reach at this point, but we are able to classify all finite nilpotent PKN-groups.

Theorem 3.10. Let $G$ be a finite nilpotent group. Then $G$ is a PKN-group if and only if its center $Z(G)$ is cyclic.

Proof. If $G$ is a PKN-group, then $Z(G)$ is cyclic by Proposition 3.1. So assume that $G$ is a finite nilpotent group with cyclic center. Then $G$ is isomorphic to the direct product of its Sylow subgroups, $G \cong \prod_{i=1}^{k} G_{i}$, where each $G_{i}$ is a $p_{i}$-group with cyclic center. By Proposition 2.5(ii), $G$ is a PKNgroup if and only if each $G_{i}$ is a PKN-group.

So we may assume without loss of generality that $G$ is a $p$-group with cyclic center $Z(G)$. But then the unique subgroup $A$ of $Z(G)$ of order $p$ is in fact the unique minimal normal subgroup of $G$. By Proposition 3.5, $G$ is a PKN-group (in fact an elementary PKN-group).

Corollary 3.11. Let $G$ be a finite nilpotent PKN-group. Then

(i) $G$ is the direct product of elementary PKN-groups;

(ii) $S(N(G))$ is a sublattice of $N(G)$. 
Proof. Part (i) follows from the fact that a PKN-p-group is elementary PKN; part (ii) then follows from Proposition 3.8.

\section{$4 \quad \mathrm{PKN}^{*}$-groups}

As we have observed in the beginning of the previous section, the class of PKN-groups is not closed under (normal) subgroups. It therefore makes sense to introduce the following class of groups.

Definition 4.1. A group $G$ is called a $P K N^{*}$-group if all of its subgroups are PKN-groups.

Our goal in this section is to classify finite $\mathrm{PKN}^{*}$-groups.

Theorem 4.2. Let $G$ be a finite group. Then the following are equivalent.

(a) $G$ is a $P K N^{*}$-group;

(b) for each prime divisor p of $|G|$, every Sylow p-subgroup of $G$ has a unique subgroup of order $p$;

(c) all Sylow subgroups of $G$ of odd order are cyclic and all Sylow 2-subgroups of $G$ are cyclic or generalized quaternion.

Proof. The fact that (b) and (c) are equivalent is well known; see, for example, [1, Chapter 8, Exercise 4]. So it suffices to show that (a) and (b) are equivalent.

Assume first that $G$ is a $\mathrm{PKN}^{*}$-group; then every subgroup of order $p^{2}$ (with $p$ prime) is itself a PKN-group, and hence such a subgroup is cyclic by Lemma 2.3(iii). As in the proof of Corollary 2.4, it follows that every Sylow $p$-subgroup has a unique subgroup of order $p$. This shows that (a) implies (b).

Conversely, suppose that $G$ is a finite group such that every Sylow $p$ subgroup of $G$ has a unique subgroup of order $p$. We claim that for every two normal subgroups $U, V \unlhd G$, the equivalence

$$
U \cap V=1 \Longleftrightarrow \operatorname{gcd}(|U|,|V|)=1
$$

holds. Indeed, if $\operatorname{gcd}(|U|,|V|)=1$, then of course $U \cap V=1$. So assume $\operatorname{gcd}(|U|,|V|) \neq 1$, then there is a prime $p$ dividing both $|U|$ and $|V|$. Let $P$ 
be a Sylow $p$-subgroup of $G$, and let $A$ and $B$ be Sylow $p$-subgroups of $U$ and $V$, respectively. Then there exist $x, y \in G$ such that $1 \neq A^{x} \leq P$ and $1 \neq B^{y} \leq P$. Since $P$ has exactly one subgroup of order $p$, this implies that $A^{x} \cap B^{y} \neq 1$ and so $U \cap V=U^{x} \cap V^{y} \neq 1$. This proves the equivalence (4.1).

Now, let $N$ be an arbitrary normal subgroup of $G$, and let $U$ be the largest normal subgroup of $G$ with order coprime to $|N|$. (Such a $U$ exists, since if $U_{1}$ and $U_{2}$ are normal subgroups with order coprime to $|N|$, then so is $U_{1} U_{2}$.) Then of course $N \cap U=1$, and by (4.1), every subgroup of $G$ which intersects $N$ trivially, has order coprime to $|N|$ and is therefore contained in $U$. Hence $U$ is a pseudocomplement of $N$ in $G$, and we conclude that $G$ is a PKN-group. Since the condition (b) is inherited to subgroups, it follows that every subgroup of $G$ is a PKN-group as well, and hence $G$ is a $\mathrm{PKN}^{*}$-group. This shows that (b) implies (a).

Remark 4.3. The finite groups with all Sylow subgroups cyclic, are always solvable, and these groups have, in fact, a very precise structure. They are known as ZM-groups; see also Definition 5.5 and Theorem 5.8 below. An important (lattice theoretical) property of the ZM-groups is that these groups are exactly the finite groups whose poset of conjugacy classes of subgroups forms a distributive lattice [4, Theorem A].

On the other hand, the groups satisfying condition (c) of Theorem 4.2 are not always solvable, but the non-solvable ones are also well understood, by the following deep result by Suzuki.

Theorem 4.4 (Suzuki [16]). Let $G$ be a non-solvable finite group such that every Sylow subgroup of $G$ is either cyclic or generalized quaternion. Then $G$ contains a normal subgroup $G_{1}$ such that $\left[G: G_{1}\right] \leq 2$ and $G_{1} \cong Z \times L$, where $Z$ is a solvable group whose Sylow subgroups are all cyclic, and $L \cong \mathrm{SL}(2, p)$ for some odd prime $p$.

In particular, for each prime $p$, the group $\mathrm{SL}(2, p)$ is a $\mathrm{PKN}^{*}$-group.

\section{Groups whose normal subgroup lattices are Stone lattices}

In this final section we present some results concerning finite groups $G$ for which the lattice $N(G)$ is a Stone lattice, i.e. a distributive pseudocomple- 
mented lattice such that $S(N(G))$ is a sublattice of $N(G)$. As a corollary, we will obtain a complete classification of the finite groups for which every subgroup is monolithic, i.e. has a unique minimal normal subgroup.

We mention that the structure of groups with distributive lattices of normal subgroups, the so-called DLN-groups, is not known, but there are some characterizations of these groups (see [10] or [12, §9.1]). We only mention the following theorem, which is a straightforward generalization of [10, Theorem 4.1]. (The result in loc. cit. is only given for a direct product with two factors.)

Theorem 5.1. Let $G=G_{1} \times \cdots \times G_{k}$ be a finite group. Then $G$ is a $D L N$-group if and only if each direct factor $G_{i}$ is a DLN-group and for all $i \neq j$, there are no central chief factors in $G_{i}$ and in $G_{j}$ the orders of which coincide.

We are now able to establish the following characterization of finite groups whose normal subgroup lattice is a Stone lattice.

Theorem 5.2. Let $G$ be a finite group. Then $N(G)$ is a Stone lattice if and only if $G$ is a direct product $G=G_{1} \times \cdots \times G_{k}$ of monolithic DLN-groups $G_{i}$, and for all $i \neq j$, there are no central chief factors in $G_{i}$ and in $G_{j}$ the orders of which coincide.

Proof. Assume first that $N(G)$ is a Stone lattice. Then $G$ is a PKN-group and $S(N(G)$ ) is a sublattice of $N(G)$, so by Proposition 3.8, G is a direct product of monolithic groups: $G=G_{1} \times \cdots \times G_{k}$. Since $G$ is a DLN-group, the conclusion now follows from Theorem 5.1.

Conversely, assume that $G=G_{1} \times \cdots \times G_{k}$, where each $G_{i}$ is a monolithic DLN-group, and for all $i \neq j$, there are no central chief factors in $G_{i}$ and in $G_{j}$ the orders of which coincide. Then by Theorem 5.1, $N(G)$ is a distributive lattice. We now claim that $G$ is a PKN-group. Indeed, let $N \unlhd G$. By distributivity of $N(G)$, we have $N=\prod_{i=1}^{k}\left(N \cap G_{i}\right)$. Without loss of generality, we may assume that $N \cap G_{i} \neq 1$ for $i \in\{1, \ldots, s\}$ and $N \cap G_{i}=1$ for $i \in\{s+1, \ldots, k\}$; in particular, $N \leq \prod_{i=1}^{s} G_{i}$. Let $N^{*}=\prod_{i=s+1}^{k} G_{i}$; we claim that $N^{*}$ is a pseudocomplement of $N$ in $N(G)$. Indeed, we clearly have $N \cap N^{*}=1$. Assume that $A \unlhd G$ is such that $N \cap A=1$. For each $i \in\{1, \ldots, s\}$, the group $G_{i}$ is monolithic, so since $N \cap G_{i} \neq 1$ we must have $A \cap G_{i}=1$. But then by distributivity, $A=\prod_{i=1}^{k}\left(A \cap G_{i}\right) \leq N^{*}$, which 
proves that $N^{*}$ is a pseudocomplement of $N$ as claimed. It now follows from Proposition 3.8 that $S(N(G))$ is a sublattice of $N(G)$, and hence $N(G)$ is a Stone lattice.

Remark 5.3. The structure of finite monolithic DLN-groups is not known either. This class of groups obviously includes the cyclic $p$-groups, but there exist a lot of non-cyclic groups which are monolithic DLN-groups (for example, the non-cyclic elementary PKN-groups presented in Remark 3.7). Nevertheless, it is easy to see that the cyclic $p$-groups are the only finite $p$-groups that are monolithic DLN-groups. Moreover, because a direct product of $k$ cyclic $p$-groups is a PKN-group if and only if $k=1$, the cyclic $p$-groups are also the only finite $p$-groups $G$ for which $N(G)$ is a Stone lattice.

The previous remark allows us to classify finite nilpotent groups whose normal subgroup lattice is a Stone lattice.

Corollary 5.4. Let $G$ be a finite nilpotent group. Then $N(G)$ is a Stone lattice if and only if $G$ is cyclic.

Proof. Since $G$ is nilpotent, it can be written as a direct product of its Sylow subgroups: $G=G_{1} \times \cdots \times G_{k}$. If $G$ is cyclic, then each of the groups $G_{i}$ is a cyclic $p_{i}$-group, and hence a monolithic DLN-group; it follows from Theorem 5.2 that $N(G)$ is a Stone lattice.

Conversely, assume that $N(G)$ is a Stone lattice. Since the orders of the Sylow subgroups are coprime, Theorem 5.2 implies that each of the lattices $N\left(G_{i}\right)$ is a Stone lattice; by Remark 5.3, each $G_{i}$ is cyclic. Hence $G$ is also cyclic.

We will now investigate finite groups for which the normal subgroup lattice of every subgroup is a Stone lattice. We start with a reduction; let us first recall the following definition which we have encountered in Remark 4.3.

Definition 5.5. A finite group $G$ is called a Zassenhaus metacyclic group, or ZM-group for short, if all Sylow subgroups of $G$ are cyclic.

Proposition 5.6. Let $G$ be a finite group. Then the following two properties are equivalent:

(a) $N(H)$ is a Stone lattice for all subgroups $H$ of $G$; 
(b) $G \cong G_{1} \times \cdots \times G_{k}$, where the $G_{i}$ are monolithic ZM-groups of coprime orders, with the property that $N\left(H_{i}\right)$ is a Stone lattice for all subgroups $H_{i}$ of $G_{i}$.

Proof. (a) $\Rightarrow$ (b). Let $H$ be a Sylow subgroup of $G$. Then $N(H)$ is a Stone lattice and therefore, by Corollary $5.4, H$ is cyclic. Thus $G$ is a ZMgroup. Since $N(G)$ is a Stone lattice, Theorem 5.2 implies that $G$ can be written as a direct product of monolithic DLN-groups $G_{i}, i \in$ $\{1, \ldots, k\}$. Since every subgroup of a ZM-group is itself a ZM-group, the $G_{i}$ are in fact ZM-groups. Suppose that there are $i \neq j$ such that $\left|G_{i}\right|$ and $\left|G_{j}\right|$ have a common prime divisor $p$. Then there are a subgroup $M_{i}$ or order $p$ in $G_{i}$, and a subgroup $M_{j}$ of order $p$ in $G_{j}$. By our hypothesis, the normal subgroup lattice of $H=M_{i} \times M_{j} \cong C_{p} \times C_{p}$ is a Stone lattice, a contradiction.

(b) $\Rightarrow$ (a). Let $H$ be an arbitrary subgroup of $G$. Since the direct factors $G_{i}$ have coprime orders, $H \cong H_{1} \times \cdots \times H_{k}$ for certain subgroups $H_{i} \leq G_{i}$. Clearly $H$ is a ZM-group, so by Remark 4.3 it is a DLN-group. Since $N\left(H_{i}\right)$ is a Stone lattice for each $i$, Proposition 3.8 implies that each $H_{i}$ is a direct product of elementary PKN-groups, and hence the same is true for $H$. Moreover, each $H_{i}$ is a PKN-group, and since the $H_{i}$ have coprime orders, Proposition 2.5(ii) implies that $H$ is a PKN-group. We conclude that $N(H)$ is a Stone lattice.

In view of Proposition 5.6(b), we will now digress on monolithic ZMgroups. The structure of ZM-groups has been completely determined by Zassenhaus.

Definition 5.7. A triple $(m, n, r)$ satisfying the conditions

$$
\operatorname{gcd}(m, n)=\operatorname{gcd}(m, r-1)=1 \quad \text { and } \quad r^{n} \equiv 1 \quad(\bmod m)
$$

will be called a $Z M$-triple, and the corresponding group

$$
\left\langle a, b \mid a^{m}=b^{n}=1, b^{-1} a b=a^{r}\right\rangle
$$

will be denoted by $\mathrm{ZM}(m, n, r)$.

Theorem 5.8 (Zassenhaus). Let $G$ be a ZM-group. Then there exists a $Z M$ triple $(m, n, r)$ such that $G \cong \mathrm{ZM}(m, n, r)$. We have $|G|=m n$ and $G^{\prime}=\langle a\rangle$ (so $\left|G^{\prime}\right|=m$ ), and $G / G^{\prime}$ is cyclic of order $n$.

Conversely, every group isomorphic to $\mathrm{ZM}(m, n, r)$ is a ZM-group. 
Proof. See, for example, [8, IV, Satz 2.11].

We can now determine the structure of monolithic ZM-groups.

Theorem 5.9. Let $G \cong \mathrm{ZM}(m, n, r)$ be a ZM-group. Then the following properties are equivalent:

(a) G is monolithic;

(b) either $m=1$ and $n$ is a prime power, or $m$ is a prime power and $r^{d} \not \equiv 1$ $(\bmod m)$ for all $1 \leq d<n$;

(c) either $|G|$ is a prime power, or $\left|G^{\prime}\right|$ is a prime power and $Z(G)=1$.

Proof. (a) $\Rightarrow(\mathrm{b})$. Assume that $G \cong \operatorname{ZM}(m, n, r)$ is monolithic. If $m=1$, then $G$ is cyclic of order $n$; for each prime divisor $p$ of $n, G$ has a minimal normal subgroup of order $p$; hence $n$ is a prime power.

So assume that $m \neq 1$, and let $p$ be a prime divisor of $m$. By the defining relations of $G$ in Definition 5.7, we see that every subgroup of $\langle a\rangle$ is normalized by $b$ and thus is a normal subgroup of $G$. In particular, $G$ has a minimal normal subgroup of order $p$.

Hence $m$ can have only one prime divisor, i.e. $m$ is a prime power; let $N$ be the minimal normal subgroup contained in $\langle a\rangle$. Suppose now that there is some $d<n$ such that $r^{d} \equiv 1(\bmod m)$. Since $r^{n} \equiv 1(\bmod m)$ as well, we may in fact assume that $d \mid n$; assume furthermore that $n / d$ is prime. Let $H=\left\langle b^{d}\right\rangle$; this is a subgroup of $G$ of prime order. By the defining relation $a b=b a^{r}$, we get

$$
a b^{d}=b^{d} a^{r^{d}}=b^{d} a
$$

since $r^{d} \equiv 1(\bmod m)$; this shows that $b^{d} \in Z(G)$, so in particular $H$ is a minimal normal subgroup of $G$, different from $N$. This contradicts the fact that $G$ is monolithic.

(b) $\Rightarrow(\mathrm{c})$. If $m=1$ and $n$ is a prime power, then $|G|=m n$ is a prime power. So assume that $m=\left|G^{\prime}\right|$ is a prime power and $r^{d} \not \equiv 1(\bmod m)$ for all $1 \leq d<n$. Let $g=b^{s} a^{t}$ with $0 \leq s \leq n-1$ and $0 \leq t \leq m-1$ be an arbitrary element of $G$; we compute

$$
[g, a]=a^{1-r^{s}}, \quad[g, b]=a^{t(r-1)} .
$$


Assume now that $g \in Z(G)$; then we must have $r^{s} \equiv 1(\bmod m)$ and $m \mid t(r-1)$. Our assumption $r^{d} \not \equiv 1(\bmod m)$ for all $1 \leq d<n$ implies $s=0$; the fact that $\operatorname{gcd}(m, r-1)$ and $t<m$ implies $t=0$. Hence $Z(G)=1$.

(c) $\Rightarrow($ a). If $|G|$ is a prime power, then $G$ is a cyclic group of prime power order and hence it is monolithic. So assume that $\left|G^{\prime}\right|$ is a prime power and $Z(G)=1$. Then $G^{\prime}$ has a unique subgroup $P$ of prime order, which is therefore a minimal normal subgroup of $G$. Let $N \unlhd G$ be an arbitrary non-trivial normal subgroup of $G$; it is sufficient to show that $N \cap G^{\prime} \neq 1$, since this will imply that $P \leq N$. So suppose that $N \cap G^{\prime}=1$. Then $[N, G]=1$, and hence $N \leq Z(G)$. But now $Z(G)=1$ implies $N=1$, a contradiction.

We now claim that every monolithic ZM-group has the property that the normal subgroup lattice of any subgroup is a Stone lattice. This statement is obvious when the group is cyclic of prime power order, so we only consider non-abelian ZM-groups.

Theorem 5.10. Let $G \cong \mathrm{ZM}(m, n, r)$ be a monolithic non-abelian ZM-group with $m=p^{k}$ for some prime $p$ and some $k \geq 1$. Then

(i) $n \mid p-1$;

(ii) the order of each element of $G$ is a divisor of $m$ or a divisor of $n$;

(iii) $N(H)$ is a Stone lattice for each subgroup $H$ of $G$. More precisely, every subgroup of $G$ is either cyclic or monolithic.

Proof. Let $G \cong \mathrm{ZM}(m, n, r)$ be a monolithic ZM-group with generators $a$ and $b$ as in Definition 5.7. By Theorem 5.9, $m=p^{k}$ for some prime $p$ and some number $k \geq 1$ since we assume $G$ to be non-abelian.

(i) By Theorem 5.9(b), the order of $r$ modulo $p^{k}$ is precisely $n$. In particular, $n \mid \phi\left(p^{k}\right)=p^{k-1}(p-1)$, where $\phi$ is the Euler totient function; since $\operatorname{gcd}(n, p)=1$, we have $n \mid p-1$.

(ii) By the defining relations in Definition 5.7, it is not very hard to compute, using induction on $d$, that

$$
\left.\left(b^{s} a^{t}\right)^{d}=b^{s d} a^{t\left(1+r^{s}+r^{2 s}+\cdots+r^{(d-1) s}\right.}\right),
$$


for all natural numbers $s, t, d$. Suppose that $G$ contains an element $g=b^{s} a^{t}$ of order $p q$, where $q$ is a prime dividing $n$. Then $b^{s}$ must have order $q$, hence we have $s=c \cdot n / q$ for some $c \in\{1, \ldots, q-1\}$. Let $x=r^{s}$; then the order of $x$ modulo $p^{k}$ is $q$. We have

$$
g^{q}=a^{t\left(1+x+x^{2}+\cdots+x^{(q-1)}\right)}, \quad g^{p q}=a^{t\left(1+x+x^{2}+\cdots+x^{(p q-1)}\right)} .
$$

Since $g$ has order $p q$, this implies

$$
\begin{aligned}
& t\left(1+x+x^{2}+\cdots+x^{(q-1)}\right) \not \equiv 0 \quad\left(\bmod p^{k}\right), \\
& t\left(1+x+x^{2}+\cdots+x^{(p q-1)}\right) \equiv 0 \quad\left(\bmod p^{k}\right) \text {. }
\end{aligned}
$$

Also observe that by (i), we have $q \mid p-1$, so in particular $x^{p} \equiv x$ $\left(\bmod p^{k}\right)$. Let $y=1+x+\cdots+x^{p-1} ;$ then $y(x-1)=x^{p}-1 \equiv x-1$ $\left(\bmod p^{k}\right)$, hence $p^{k} \mid(y-1)(x-1)$. However, $x \not \equiv 1\left(\bmod p^{k}\right)$, and therefore $p \mid y-1$. In particular, $\operatorname{gcd}(y, p)=1$.

Since $x^{p} \equiv x\left(\bmod p^{k}\right)$, we have

$$
\begin{aligned}
& 1+x+x^{2}+\cdots+x^{(p q-1)} \\
& =\left(1+x+x^{2}+\cdots+x^{(p-1)}\right)\left(1+x^{p}+x^{2 p}+\cdots+x^{(q-1) p}\right) \\
& \quad \equiv y\left(1+x+x^{2}+\cdots+x^{(q-1)}\right) \quad\left(\bmod p^{k}\right)
\end{aligned}
$$

since $\operatorname{gcd}(y, p)=1$, this implies the equivalence

$$
\begin{aligned}
t\left(1+x+x^{2}+\cdots+x^{(p q-1)}\right) \equiv 0 \quad\left(\bmod p^{k}\right) & \\
& \Longleftrightarrow t\left(1+x+x^{2}+\cdots+x^{(q-1)}\right) \equiv 0 \quad\left(\bmod p^{k}\right)
\end{aligned}
$$

This contradicts equations (5.2) and (5.3), and (ii) follows.

(iii) Let $H$ be an arbitrary subgroup of $G$. If $H$ is abelian, then it is cyclic, so Corollary 5.4 implies that $N(H)$ is a Stone lattice. So we may assume that $H$ is non-abelian; in particular, the derived subgroup $H^{\prime}$ is nontrivial, and since $H^{\prime} \leq G^{\prime}=\langle a\rangle$, this implies that $g=a^{p^{k-1}} \in H$, and therefore $p|| H \mid$. If $p$ were the only prime dividing $|H|$, then $H$ would be cyclic, contradicting the fact that $H$ is non-abelian; hence there is some prime $q \mid n$ with $q|| H \mid$. Let $h \in H$ be an element of order $q$.

Suppose that $Z(H) \neq 1$. If $Z(H)$ contains an element $x$ of order $p$, then $x h$ has order $p q$, contradicting (ii). If $Z(H)$ does not contain elements 
of order $p$, then it must contain an element $y$ of order $q^{\prime}$ for some prime $q^{\prime} \mid n$, but then $y g$ has order $p q^{\prime}$, again contradicting (ii). We conclude that $Z(H)=1$, and hence by Theorem $5.9, H$ is a monolithic ZM-group; in particular, $N(H)$ is a Stone lattice.

We now obtain the complete classification of all groups with the property that each subgroup has a Stone normal subgroup lattice as an easy corollary.

Theorem 5.11. Let $G$ be a finite group. Then the following two properties are equivalent:

(a) $N(H)$ is a Stone lattice for all subgroups $H$ of $G$;

(b) $G \cong G_{1} \times \cdots \times G_{k}$, where the $G_{i}$ are monolithic ZM-groups of coprime orders.

Proof. This follows immediately from Proposition 5.6 and Theorem 5.10.

We end this section by classifying all finite groups such that every subgroup is monolithic.

Theorem 5.12. Let $G$ be a finite group; then the following two properties are equivalent:

(a) every subgroup of $G$ is monolithic;

(b) either $G$ is a cyclic group of order $p^{k}$, or $G$ is a ZM-group of order $p^{k} q^{\ell}$ with $Z(G)=1$, where $p, q$ are distinct primes.

Proof. (a) $\Rightarrow(\mathrm{b})$. Assume that every subgroup of $G$ is monolithic. Then every Sylow subgroup of $G$ is cyclic, hence $G$ is a monolithic ZM-group, say $G \cong \mathrm{ZM}(m, n, r)$. Assume that $|G|$ is not a prime power; then by Theorem 5.9, $Z(G)=1$ and $m$ is a prime power $p^{k}$. On the other hand, the group $\langle b\rangle$ is a cyclic group of order $n$; such a group can only be monolithic if $n$ is a prime power $q^{\ell}$.

$(\mathrm{b}) \Rightarrow(\mathrm{a})$. If $G$ is a cyclic group of prime power order, then each of its subgroups is monolithic; so assume that $G$ is a ZM-group of order $p^{k} q^{\ell}$ with $Z(G)=1$. By Theorem $5.9, G$ is monolithic. Now let $H$ be an arbitrary subgroup of $G$. Then by Theorem 5.10 (iii), $H$ is cyclic or monolithic. If it is monolithic, we are done, so assume $H$ is cyclic. By 
Theorem 5.10(ii), the order of each element is either a power of $p$ or a power of $q$, so it follows that $H$ is a cyclic $p$-group or a cyclic $q$-group. In both cases, $H$ is monolithic.

\section{References}

[1] M. Aschbacher, "Finite group theory", Second edition, Cambridge Studies in Advanced Mathematics 10, Cambridge University Press, Cambridge, 2000.

[2] R. Baer, The Significance of the System of Subgroups for the Structure of the Group, Amer. J. Math. 61 (1939), no. 1, 1-44.

[3] G. Birkhoff, "Lattice theory", Amer. Math. Soc., Providence, R.I., 1967.

[4] R. Brandl, G. Cutolo and S. Rinauro, Posets of subgroups of groups and distributivity, Boll. U.M.I. (7) 9-A (1995), 217-223.

[5] Gr. G. Călugăreanu, Abelian groups with pseudocomplemented lattice of subgroups, Studia Univ. Babeş-Bolyai Cluj-Napoca (3) 31 (1986), 3941 .

[6] V. Glivenko, Sur quelques points de la logique de M. Brouwer, Bull. Acad. Sc. Belgique 15 (1929), 183-188.

[7] G. Grätzer, "General lattice theory", Academic Press, New York, 1978.

[8] B. Huppert, "Endliche Gruppen I", Die Grundlehren der Mathematischen Wissenschaften, Band 134, Springer-Verlag, Berlin, New York, 1967.

[9] O. Ore, Structures and group theory II, Duke Math. J. 4 (1938), 247269.

[10] G. Pazderski, On groups for which the lattice of normal subgroups is distributive, Beiträge zur Algebra und Geometrie 24 (1987), 185-200.

[11] A. Rottlaender, Nachweis der Existenz nicht-isomorpher Gruppen von gleicher Situation der Untergruppen, Math. Z. 28 (1928), no. 1, 641-653. 
[12] R. Schmidt, "Subgroup lattices of groups", de Gruyter Expositions in Mathematics 14, de Gruyter, Berlin, 1994.

[13] R. Schmidt, New results and methods in the theory of subgroup lattices of groups, Quaderni di Mat. 8 (Topics in Infinite Groups), Eds. M. Curzio, F. De Giovanni, 2000, 239-275.

[14] M. Suzuki, On the lattice of subgroups of finite groups, Trans. Amer. Math. Soc. 70 (1951), 345-371.

[15] M. Suzuki, On the L-homomorphisms of finite groups, Trans. Amer. Math. Soc. 70 (1951), 372-386.

[16] M. Suzuki, On finite groups with cyclic Sylow subgroups for all odd primes, Amer. J. Math. 77 (1955), 657-691.

[17] M. Suzuki, "Structure of a group and the structure of its lattice of subgroups", Ergebnisse der Mathematik und ihrer Grenzgebiete, Neue Folge, Heft 10, Springer-Verlag, Berlin-Göttingen-Heidelberg, 1956.

[18] M. Suzuki, "Group theory", I, II, Springer-Verlag, Berlin, 1982, 1986.

[19] M. Tărnăuceanu, On groups whose lattices of subgroups are pseudocomplemented, Fuzzy Systems \& Artificial Intelligence (2) 10 (2004), 45-49.

[20] M. Tărnăuceanu, Pseudocomplemented groups, An. Univ. "Al.I. Cuza", Iaşi, Math., (1) 51 (2005), 201-205.

[21] M. Tărnăuceanu, Complementation in subgroup lattices, Sci. Ann. Univ. Agric. Sci. Vet. Med. 49 (2006), 285-303.

[22] M. Tărnăuceanu, Complementation in normal subgroup lattices, Sci. Ann. Univ. Agric. Sci. Vet. Med. 49 (2006), 305-322.

[23] M. Tărnăuceanu, "Groups determined by posets of subgroups", Ed. Matrix Rom, Bucureşti, 2006.

[24] K. H. Toh, Groups with normal subgroups possessing subnormal complements, Proc. Amer. Math. Soc. (2) 41 (1973), 378-380. 
[25] G. Whitson, Finite groups whose subgroup, composition subgroup, or normal subgroup lattice is an ortholattice, Algebra Universalis 8 (1978), $123-127$.

[26] J. Wiegold, On direct factors in groups, J. London Math. Soc. 35 (1960), 310-320.

Tom De Medts

Department of Pure Mathematics and Computer Algebra

Ghent University

Galglaan 2, B-9000 Gent, Belgium

e-mail: tdemedts@cage.UGent.be
Marius Tărnăuceanu

Faculty of Mathematics

"Al.I. Cuza" University

Iaşi, Romania

e-mail: tarnauc@uaic.ro 